\title{
Optimal Online Two-way Trading with Bounded Number of Transactions
}

\author{
Stanley P. Y. Fung \\ Department of Informatics, University of Leicester, Leicester LE1 7RH, \\ United Kingdom. pyf1@leicester.ac.uk
}

\begin{abstract}
We consider a two-way trading problem, where investors buy and sell a stock whose price moves within a certain range. Naturally they want to maximize their profit. Investors can perform up to $k$ trades, where each trade must involve the full amount. We give optimal algorithms for three different models which differ in the knowledge of how the price fluctuates. In the first model, there are global minimum and maximum bounds $m$ and $M$. We first show an optimal lower bound of $\varphi$ (where $\varphi=M / m$ ) on the competitive ratio for one trade, which is the bound achieved by trivial algorithms. Perhaps surprisingly, when we consider more than one trade, we can give a better algorithm that loses only a factor of $\varphi^{2 / 3}$ (rather than $\varphi$ ) per additional trade. Specifically, for $k$ trades the algorithm has competitive ratio $\varphi^{(2 k+1) / 3}$. Furthermore we show that this ratio is the best possible by giving a matching lower bound. In the second model, $m$ and $M$ are not known in advance, and just $\varphi$ is known. We show that this only costs us an extra factor of $\varphi^{1 / 3}$, i.e., both upper and lower bounds become $\varphi^{(2 k+2) / 3}$. Finally, we consider the bounded daily return model where instead of a global limit, the fluctuation from one day to the next is bounded, and again we give optimal algorithms, and interestingly one of them resembles common trading algorithms that involve stop loss limits.
\end{abstract}

\section{Introduction}

The model. We consider a scenario commonly faced by investors. The price of a stock varies over time. In this paper we use a 'day' as the smallest unit of time, so there is one new price each day. Let $p(i)$ be the price at day $i$. The investor has some initial amount of money. Over a time horizon of finite duration, the investor wants to make a bounded number of trades of this one stock. Each trade $(b, s)$ consists of a buy transaction at day $b$, followed by a sell transaction at day $s$ where $s>b$. (Thus one trade consists of two transactions.) Both transactions are 'all-in': when buying, the investor uses all the money available, and when selling all stock they currently own is sold. A sale must be made before the next purchase can take place. Also, no short selling is allowed, i.e., there can be no selling if the investor is not currently holding stock. When the end of the time horizon is reached, i.e., on the last day, no buying is allowed and the investor must sell off all the stocks that they still hold back to cash at the price of the day. 
There are a number of rationales for considering a bounded number of trades and/or that trades must involve all the money available. Individual, amateur investors typically do not want to make frequent transactions due to high transaction fees. Often transaction fees have a fixed component (i.e., a fixed amount or a minimum tariff per transaction, irrespective of the trading amount) which makes transaction fees disproportionally high for small trades. Frequent trading also requires constant monitoring of the markets which amateur investors may not have the time or resources for; often they only want to change their investment portfolios every now and then. Also, for investors with little money available, it is not feasible or sensible to divide them into smaller pots of money, in arbitrary fractions as required by some algorithms. The finiteness of the time horizon (and that its length is possibly unknown as well) corresponds to situations where an investor may be forced to sell and leave the market due to unexpected need for money elsewhere, for example.

Each trade with a buying price of $p(b)$ and a selling price of $p(s)$ gives a gain of $p(s) / p(b)$. This represents how much the investor has after the trade if they invested 1 dollar in the beginning. Note that this is a ratio, and can be less than 1 , meaning there is a loss, but we will still refer to it as a 'gain'. If a series of trades are made, the overall gain or the return of the algorithm is the product of the gains of each of the individual trades. This correctly reflects the fact that all the money after each trade is re-invested in the next.

Since investors make decisions without knowing future stock prices, the problem is online in nature. We measure the performance of online algorithms with competitive analysis, i.e., by comparing it with the optimal offline algorithm OPT that knows the price sequence in advance and can therefore make optimal decisions. The competitive ratio of an online algorithm ONL is the worst possible ratio of the return of OPT to the return of ONL, over all possible input (price) sequences. The multiplicative nature of the definition of the return (instead of specifying a negative value for a loss) means that the competitive ratio can be computed in the normal way in the case of a loss: for example, if OPT makes a gain of 2 and ONL makes a 'gain' of $1 / 3$, then the competitive ratio is 6 .

Three models on the knowledge of the online algorithm. We consider three different models on how the price changes, or equivalently, what knowledge the online algorithm has in advance. In the first model, the stock prices are always within a range $[m . . M]$, i.e., $m$ is the minimum possible price and $M$ the maximum possible price. Both $m$ and $M$ are known to the online algorithm up front. In the second model, the prices still fluctuate within this range, but $m$ and $M$ are not (initially) known; instead only their ratio $\varphi=M / m$, called the fluctuation ratio, is known. In both these models the length of the time horizon (number of days) is unknown (until the final day arrives). In the third model, called the bounded daily return model, there is no global minimum or maximum price. Instead, the maximum fluctuation from day to day is bounded: namely, the price $p(i+1)$ of the next day is bounded by the price $p(i)$ of the current day by $p(i) / \beta \leq p(i+1) \leq \alpha p(i)$ for some $\alpha, \beta>1$. This means the prices cannot suddenly change a lot. Many stock markets implement the so-called 'circuit 
breakers' where trading is stopped when such limits are reached. Here $\alpha, \beta$ and the trade duration $T$ are known to the online algorithm. All three models are well-established in the algorithms literature; see e.g. [1,5].

Previous results and related work. Financial trading and related problems are obviously important topics and have been much studied from the online algorithms perspective. A comprehensive survey is given in [8]. Here we only sample some of the more important results and those closer to the problems we study here. In the one-way search problem, the online player chooses one moment of time to make a single transaction from one currency to another currency. Its return is simply the price at which the transaction takes place. A reservation price (RP) based policy is to buy as soon as the price reaches or goes above a pre-set reservation price. It is well-known that, if $m$ and $M$ are known, the RP policy with a reservation price of $\sqrt{\mathrm{Mm}}$ is optimal and achieves a competitive ratio of $\sqrt{\varphi}$. If only $\varphi$ is known, then no deterministic algorithm can achieve a ratio better than $\varphi$. With the help of randomization, however, a random mix of different RPs gives a competitive ratio of $O(\log \varphi)$ if $\varphi$ is known. Even if $\varphi$ is not known, a competitive ratio of $O\left(\log \varphi \cdot \log ^{1+\epsilon}(\log \varphi)\right)$ can be achieved. See [5] for all the above results and more discussions.

In the one-way trading problem, the objective is again to maximize the return in the other currency, but there can be multiple transactions, i.e., not all the money has to be traded in one go. (This distinction of terminology between search and trading is used in [5], but is called non-preemptive vs. preemptive in [8]. We prefer calling them unsplittable vs. splittable here.) The relation between one-way trading and randomized algorithms for one-way search is described in [5]. Many variations of one-way search or one-way trading problems have since been studied; some examples include the bounded daily return model $[1,11]$, searching for $k$ minima/maxima instead of one [7], time-varying bounds [4], unbounded prices [2], search with advice complexity [3], etc.

What we study here, however, is a two-way version of the unsplittable trading problem $^{1}$, which is far less studied. Here the online player has to first convert from one currency (say cash) to another (a stock), hopefully at a low price, and then convert back from the stock to cash at some later point, hopefully at a high price. All the money must be converted back to the first currency when or before the game ends. This model is relevant where investors are only interested in short term, speculative gains. For the models with known $m, M$ or known $\varphi$ and with one trade, Schmidt et al. [9] gave a $\varphi$-competitive algorithm; it uses the same RP for buying and selling. But consider the Do-Nothing algorithm that makes no trades at all. Clearly it is also $\varphi$-competitive as ONL's gain is 1 and OPT's gain is at most $\varphi$ (if the price goes from $m$ to $M$ ). A number of common algorithms, such as those based on moving averages, were also studied in [8]. It was shown that they are $\varphi^{2}$-competitive (and not better), which are therefore even worse. It is easy to show that these algorithms have competitive

\footnotetext{
${ }^{1}$ In the terminology of [5] this should be called 'two-way search', but we feel that the term does not convey its application in stock market trading.
} 
ratios $\varphi^{k}$ and $\varphi^{2 k}$ respectively when extended to $k$ trades. Schroeder et al. [10] gave some algorithms for the bounded daily return model, without limits on the number of trades. However, most of these algorithms tend to make decisions that are clearly bad, have the worst possible performance (like losing by the largest possible factor every day throughout), and have competitive ratios no better than what is given by Do-Nothing.

Our results. In this paper we consider the two-way unsplittable trading problem where a bounded number $k$ of trades are permitted, and derive optimal algorithms. First we consider the model with known $m$ and $M$. We begin by considering the case of $k=1$. Although some naive algorithms are known to be $\varphi$-competitive and seemingly nothing better is possible, we are not aware of any matching general lower bound. We give a general lower bound of $\varphi$, showing that the naive algorithms cannot be improved. The result is also needed in subsequent lower bound proofs.

It may be tempting to believe that nothing can beat the naive algorithm also for more trades. Interestingly, we prove that for $k \geq 2$ this is not true. While naive algorithms like Do-Nothing are no better than $\varphi^{k}$-competitive, we show that a reservation price-based algorithm is $\varphi^{(2 k+1) / 3}$-competitive. For example, when $k=2$, it is $\varphi^{5 / 3}$-competitive instead of trivially $\varphi^{2}$-competitive. Furthermore, we prove a matching lower bound, showing that the algorithm is optimal.

Next, we consider the model where only $\varphi$ is known, and give an algorithm with a competitive ratio of $\varphi^{(2 k+2) / 3}$, i.e., only a factor $\varphi^{1 / 3}$ worse than that of the preceding model. Again we show that this bound is optimal.

Finally we consider the bounded daily return model, and give two optimal algorithms where the competitive ratio depends on $\alpha, \beta$ and $T$. For example, with one trade and in the symmetric case $\alpha=\beta$, the competitive ratio is $\alpha^{2 T / 3}$. While this is exponential in $T$ (which is unavoidable), naive algorithms could lose up to a factor of $\max (\alpha, \beta)$ every day, and Do-Nothing has a competitive ratio of $\alpha^{T}$. One of the algorithms uses the 'stop loss / lock profit' strategy commonly used in real trading; as far as we are aware, this is the first time where competitive analysis justifies this common stock market trading strategy, and in fact suggests what the stop loss limit should be.

Some proofs are omitted due to space constraints.

\section{Known $m$ and $M$}

In this section, where we consider the model with known $m$ and $M$, we can without loss of generality assume that $m=1$. This is what we will do to simplify notations. It also means $M$ and $\varphi$ are equal and are sometimes used interchangeably.

Theorem 1. For $k=1$, no deterministic algorithm has a competitive ratio better than $\varphi^{1-\epsilon}$, for any $\epsilon>0$. 
Proof. Choose $n=\lceil 1 / \epsilon\rceil$ and define $v_{i}=M^{i / n}$ for $i=0,1, \ldots, n$. The following price sequence is released until ONL buys: $v_{n-1}, M, v_{n-2}, M, \ldots, v_{i}, M, \ldots, v_{1}$, $M, v_{0}$. If ONL does not buy at any point, or buys at price $M$, then its return is at most 1 . Then OPT buys at $v_{1}$ and sells at $M$ to get a return of $M^{1-1 / n}$. So suppose ONL buys at $v_{i}$ for some $1 \leq i \leq n-1$. (It cannot buy at $v_{0}$ as it is the last time step.) As soon as ONL bought, the rest of the sequence is not released; instead the price drops to $m$ and the game ends. ONL's return is $m / v_{i}$. If $i=n-1$, then OPT makes no trade and its return is 1 , so competitive ratio $=v_{n-1} / m=M^{1-1 / n}$. Otherwise, if $i<n-1$, OPT buys at $v_{i+1}$ (two days before ONL's purchase) and sells at the next day at price $M$, giving a return of $M / v_{i+1}$. The competitive ratio is therefore $M v_{i} /\left(m v_{i+1}\right)=M^{1-1 / n}$.

Thus in all cases the competitive ratio is at least $M^{1-1 / n} \geq \varphi^{1-\epsilon}$.

Note that the proof does not require ONL to use only one trade: it cannot benefit even if it is allowed to use more trades. This fact will be used later in Theorems 3 and 5 .

For $k>1$, we analyze the following algorithm:

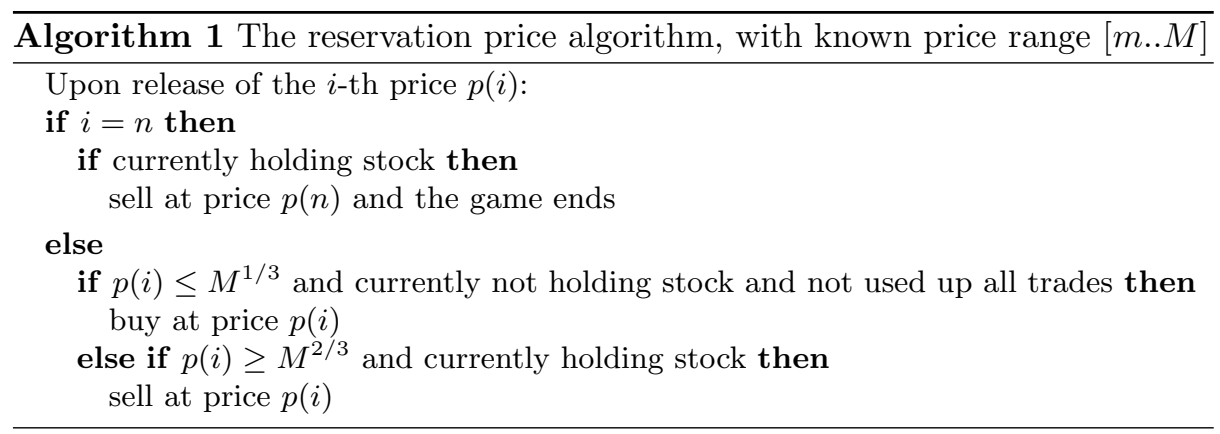

Theorem 2. Algorithm 1 has competitive ratio $\varphi^{(2 k+1) / 3}$, for $k \geq 1$.

Proof. First we make a few observations. We call a trade winning if its gain is higher than 1, and losing otherwise. Any winning trade made by ONL has a gain of at least $M^{1 / 3}$. If the algorithm makes a losing trade, it must be a forced sale at the end and the gain is not worse than $M^{-1 / 3}$. Moreover, it follows that the algorithm cannot trade anymore after it.

We consider a number of cases separately based on the sequence of win/loss trades. If we use $W$ and $L$ to denote a winning and a losing trade respectively, then following the above discussion, the possible cases are: nil (no trade), $L$, $W^{j} L$ for $1 \leq j \leq k-1$, and $W^{j}$ for $1 \leq j \leq k$. (Here $W^{j}$ denotes a sequence of $j$ consecutive $W$ 's.)

Case nil: Since ONL has never bought, the prices were never at or below $M^{1 / 3}$ (except possibly the last one, but neither OPT nor ONL can buy there) and 

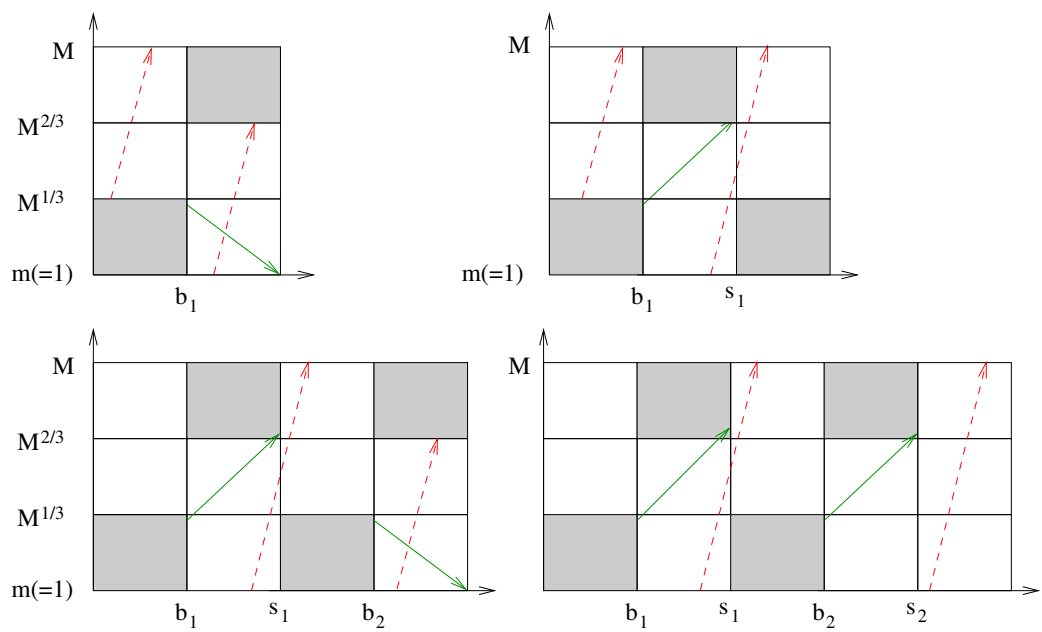

Fig. 1. Four cases illustrated, for $k=2$. Horizontal axis is time, vertical axis is price. Shaded regions are the regions where the prices cannot fall into. Green solid arrows depict possible buying and selling actions of ONL, red dashed arrows for OPT. Top left: case L, Top right: case W, Bottom left: case WL, Bottom right: case WW.

hence OPT's return cannot be better than $\left(M / M^{1 / 3}\right)^{k}=M^{2 k / 3}$. ONL's return is 1 . So the competitive ratio is at most $M^{2 k / 3}$.

Case $L$ : Suppose ONL buys at time $b_{1}$ and is forced to sell at the end. The prices before $b_{1}$ cannot be lower than $M^{1 / 3}$ (or else it would have bought) and the prices after $b_{1}$ cannot be higher than $M^{2 / 3}$ (or else it would have sold). Thus, it is easy to see (Figure 1) that OPT cannot make any trade with gain higher than $M^{2 / 3}$. So the competitive ratio is at most $\left(M^{2 / 3}\right)^{k} / M^{-1 / 3}=M^{(2 k+1) / 3}$.

Case $W$ : Suppose ONL buys at time $b_{1}$ and sells at time $s_{1}$. Then before $b_{1}$, the prices cannot be below $M^{1 / 3}$; between $b_{1}$ and $s_{1}$, the prices cannot be higher than $M^{2 / 3}$; and after $s_{1}$, the prices cannot be lower than $M^{1 / 3}$. It can be seen from Figure 1 that OPT can make at most one trade with gain $M$ (crossing time $s_{1}$ ); any other trade it makes must be of gain at most $M^{2 / 3}$. So the competitive ratio is at most $M\left(M^{2 / 3}\right)^{k-1} / M^{1 / 3}=M^{2 k / 3}$.

Case $W^{j} L, 1 \leq j \leq k-1$ : Similarly, we can partition the timeline into regions (Figure 1), from which we can see that OPT can make at most $j$ trades of gain $M$ and the rest have gain at most $M^{2 / 3}$. Thus competitive ratio = $\left(M^{j}\left(M^{2 / 3}\right)^{k-j}\right) /\left(\left(M^{1 / 3}\right)^{j} M^{-1 / 3}\right)=M^{(2 k+1) / 3}$.

Case $W^{j}, 1<j \leq k-1$ : This can only be better than the previous case, as OPT again can make at most $j$ trades of gain $M$ and the rest have gain at most $M^{2 / 3}$, but ONL's return is better than the previous case.

Case $W^{k}$ : In this case the competitive ratio is simply $M^{k} /\left(M^{1 / 3}\right)^{k}=M^{2 k / 3}$. 
Theorem 3. No deterministic algorithm has a competitive ratio better than $\varphi^{(2 k+1) / 3-\epsilon}$, for any $\epsilon>0$ and $k \geq 1$.

Proof. The prices are released in up to $k$ rounds. The final round $k$ is a special round. For all other rounds, we maintain the following invariants. For each $1 \leq$ $i \leq k-1$, just before the $i$-th round starts, OPT completed exactly $i-1$ trades, is holding no stock, and accumulated a return of exactly $M^{i-1}$, while ONL completed at most $i-1$ trades, is holding no stock, and accumulated a return of at most $M^{(i-1) / 3}$. So the competitive ratio up to this point is at least $M^{2(i-1) / 3}$.

For any $i<k$, round $i$ begins with the price sequence $M^{1 / 3}, M, M^{1 / 3}, M, \ldots$ until either ONL buys or $k-i$ such pairs of oscillating prices have been released. If ONL does not buy at any point, then the round ends. Clearly, ONL maintains its variants. OPT makes $k-i$ trades giving a total gain of $\left(M^{2 / 3}\right)^{k-i}$ in this round, and thus the accumulated competitive ratio is $M^{2(k-1) / 3}$. It also used $(i-1)+(k-i)=k-1$ trades. Any remaining intermediate rounds are then skipped and we jump directly to the special last round $k$.

Otherwise, assume ONL buys at one of the $M^{1 / 3}$ prices ( $M$ is clearly even worse). The rest of that sequence will not be released. Instead, the price sequence that follows is $m, M^{2 / 3}, m, M^{2 / 3}, \ldots$ until either ONL sells or $k-i+1$ such pairs of oscillating prices were released. If ONL does not sell at any of these, then the price drops to $m$ and the game ends (with no further rounds, not even the special round). ONL's gain in this round is $M^{-1 / 3}$. OPT uses all its remaining $k-i+1$ trades and gains $\left(M^{2 / 3}\right)^{k-i+1}$. Combining with the previous rounds, the competitive ratio is at most $M^{2(i-1) / 3} M^{2(k-i+1) / 3} / M^{-1 / 3}=M^{(2 k+1) / 3}$.

Otherwise ONL sells at one of the $M^{2 / 3}$ prices ( $m$ is even worse). The rest of that sequence will not be released; instead the price goes up to $M$ and this round ends. OPT's gain in this round is $M$ by making one trade from $m$ to $M$; ONL gains $M^{1 / 3}$. Thus the invariants are maintained and we move on to the next round. (Regarding the invariant that ONL is not holding stock at the end of the round, we can assume w.l.o.g. that ONL does not buy at the last price $M$, since clearly it cannot make a profit doing so. In any case, even if it does buy, it can be treated as if it were buying at the beginning of the next round.)

Finally, if we arrive at round $k$, then the same price sequence as in Theorem 1 is used to give an additional factor of $M^{1-\epsilon}$ to the competitive ratio. Note that at the start of this round, OPT has one trade left, and ONL has one or more trades left, but that will not help. Thus the competitive ratio is not better than $M^{2(k-1) / 3+1-\epsilon}=M^{(2 k+1) / 3-\epsilon}$.

\section{Known $\varphi$ only}

For $k=1$ Do-Nothing is clearly still $\varphi$-competitive, and Theorem 1 still applies here, so we focus on $k>1$. We adapt Algorithm 1 by buying only when it is certainly 'safe', i.e., when it is certain that the price is within the lowest $\varphi^{1 / 3}$ of the actual price range, and sells when it gains $\varphi^{1 / 3}$. The formal description is given in Algorithm 2. Let $M_{t}$ be the maximum price observed up to and including day $t$. Note that $M_{t}$ is a stepwise increasing function of $t$. 


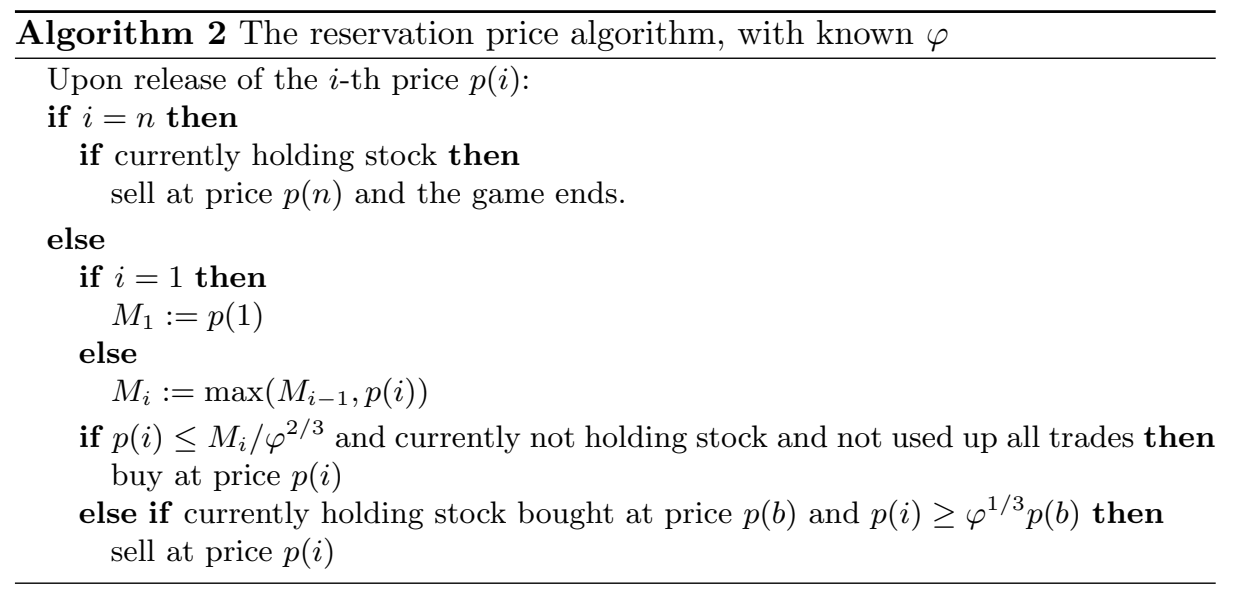

Theorem 4. Algorithm 2 has competitive ratio $\varphi^{(2 k+2) / 3}$, for any $k \geq 2$.

Proof. Clearly ONL gets the same as in Theorem 2: each winning trade has gain at least $\varphi^{1 / 3}$ and a losing trade, which can only appear as the last trade, has gain at least $\varphi^{-1 / 3}$. The difference is in how we bound OPT's gain.

In the case of $W^{k}$ (ONL makes $k$ winning trades) then the same argument as Theorem 2 applies, so in the following we only consider the case where ONL did not use up all its trade, i.e., it is always able to buy if it is not holding.

A sell event happens at a day when ONL sells and makes a profit (i.e., excludes the forced sale at the end). An $M$-change event happens at day $t$ when $M_{t}$ changes. Each OPT trade $\left(b^{*}, s^{*}\right)$ can be classified into one of the following types:

(1) There is at least one sell event during $\left[b^{*}, s^{*}\right]$. Clearly the number of such OPT trades is limited by the number of sell events. Each such trade can gain up to $\varphi$.

(2) There is no sell event during $\left[b^{*}, s^{*}\right]$, and at $b^{*}$ ONL is holding or buying. Suppose ONL's most recent purchase is at time $b \leq b^{*}$. Then $p(b) \leq M_{b} / \varphi^{2 / 3} \leq$ $M_{b^{*}} / \varphi^{2 / 3}$. It is holding stock throughout and still did not sell at $s^{*}$ (or is forced to sell if $s^{*}$ is the last day), hence $p\left(s^{*}\right)<p(b) \varphi^{1 / 3} \leq M_{b^{*}} / \varphi^{1 / 3}$. But clearly $p\left(b^{*}\right) \geq M_{b^{*}} / \varphi$, hence the gain of OPT is at most $\varphi^{2 / 3}$.

(3) There is no sell event during $\left[b^{*}, s^{*}\right]$, at $b^{*}$ ONL is neither holding nor buying, and there is no M-change event in $\left(b^{*}, s^{*}\right]$. We have $p\left(b^{*}\right)>M_{b^{*}} / \varphi^{2 / 3}$ as otherwise ONL would have bought at $b^{*}$. Clearly $p\left(s^{*}\right) \leq M_{s^{*}}=M_{b^{*}}$. Hence the gain of OPT is $p\left(s^{*}\right) / p\left(b^{*}\right)<\varphi^{2 / 3}$.

(4) There is no sell event during $\left[b^{*}, s^{*}\right]$, at $b^{*}$ ONL is neither holding nor buying, and there is/are M-change event(s) in $\left(b^{*}, s^{*}\right]$. Suppose there are a total of $x$ such OPT trades, $\left(b_{1}^{*}, s_{1}^{*}\right),\left(b_{2}^{*}, s_{2}^{*}\right), \ldots,\left(b_{x}^{*}, s_{x}^{*}\right)$, in chronological order. Note that $p\left(b_{i}^{*}\right)>M_{b_{i}^{*}} / \varphi^{2 / 3}$ or else ONL would have bought at $b_{i}^{*}$. So for all $i, p\left(b_{i+1}^{*}\right)>M_{b_{i+1}^{*}} / \varphi^{2 / 3} \geq M_{s_{i}^{*}} / \varphi^{2 / 3} \geq p\left(s_{i}^{*}\right) / \varphi^{2 / 3}$. Thus the total gain of 
these $x$ trades is

$$
\prod_{i=1}^{x} \frac{p\left(s_{i}^{*}\right)}{p\left(b_{i}^{*}\right)}=\frac{1}{p\left(b_{1}^{*}\right)} \frac{p\left(s_{1}^{*}\right)}{p\left(b_{2}^{*}\right)} \cdots \frac{p\left(s_{x-1}^{*}\right)}{p\left(b_{x}^{*}\right)} \frac{p\left(s_{x}^{*}\right)}{1}<\frac{p\left(s_{x}^{*}\right)}{p\left(b_{1}^{*}\right)}\left(\varphi^{2 / 3}\right)^{x-1} \leq \varphi^{(2 x+1) / 3} .
$$

Suppose ONL makes $y$ winning trades and one losing trade. Then OPT makes at most $y$ trades of type (1), gaining at most $\varphi^{y}$ from those. Then, if $x$ of OPT's trades are of type (4), they in total gives another gain of at most $\varphi^{(2 x+1) / 3}$. The remaining trades are of types $(2)$ and (3), gaining $\varphi^{2 / 3}$ each. The competitive ratio is therefore at most

$$
\frac{\varphi^{y} \varphi^{(2 x+1) / 3} \varphi^{2(k-x-y) / 3}}{\varphi^{y / 3} \varphi^{-1 / 3}}=\varphi^{(2 k+2) / 3} .
$$

If ONL makes $y<k$ winning trades and no losing trade, the competitive ratio can only be better, as OPT's return is as above but ONL's is $\varphi^{1 / 3}$ better.

Theorem 5. No deterministic algorithm is better than $\varphi^{(2 k+2) / 3-\epsilon}$-competitive, for any $\epsilon>0$ and $k \geq 2$.

Proof. Again there will be a number of rounds. Round 1 is special, in that OPT will get a factor of $\varphi$ better than ONL but will afterwards reveal knowledge of $m$ and $M$. Rounds 2 to $k$ are then similar to Theorem 3 .

Round 1: The first price is 1 . If ONL does not buy, then the price goes up to $\varphi$. OPT makes one trade and gains $\varphi$. Now we know the range is $[1 . . \varphi]$, and we can assume w.l.o.g. that ONL does not buy at $\varphi$. Then the round ends. At the end of this round, both OPT and ONL are not holding stock, OPT made one trade and ONL none, but ONL is a factor of $\varphi$ behind in the return.

Otherwise, if ONL buys at 1 , then the subsequent price sequence is $1 / \varphi, 1$, $1 / \varphi, 1, \ldots$ for up to $k$ such pairs, until ONL sells. Now we know the range is $[1 / \varphi . .1]$. Without loss of generality we can assume ONL does not sell at $1 / \varphi$ since it is clearly the lowest possible price. If ONL does not sell at any point, then the game ends with no further rounds. OPT makes $k$ trades gaining $\varphi^{k}$, and ONL's gain is 1 . The competitive ratio is $\varphi^{k}$, which is at least $\varphi^{(2 k+2) / 3}$. If ONL sells at some point with price 1 , then the sequence stops and this round ends. OPT buys at $1 / \varphi$ and sells at 1 , getting a gain of $\varphi$. ONL's gain is 1 . Both OPT and ONL used one trade, and OPT is a factor of $\varphi$ ahead of ONL.

Each of rounds 2 to $k-1$ are the same as the intermediate rounds in Theorem 3, with OPT gaining a factor of $\varphi^{2 / 3}$ ahead of ONL in each round.

Finally, in round $k$ we use the same price sequence in Theorem 1 , which gives an extra factor of $\varphi^{1-\epsilon}$. Note that ONL may have more trades left then OPT (in addition to the same reason as in Theorem 3, in round 1 ONL may have done no trade), but again it is not useful for ONL.

\section{Bounded daily return, known duration}

Recall that in this model, the prices are bounded by $p(i) / \beta \leq p(i+1) \leq \alpha p(i)$ for some $\alpha, \beta>1$. Trades can take place at days $0,1, \ldots, T$. 
Theorem 6. No deterministic algorithm has a competitive ratio better than $\alpha^{T(2 k \log \beta) /((k+1) \log \beta+k \log \alpha)}$.

Proof. The adversary strategy is very simple and natural: whenever ONL is not holding stock, the price goes up by a factor of $\alpha$ every day, and while it is holding stock it goes down by $\beta$ every day. Let the ONL trades be $\left(b_{i}, s_{i}\right), i=1, \ldots, k$. (If there are fewer than $k$ trades, auxillary ones with $b_{i}=s_{i}$ can be added.) Define $t_{2 i-1}=b_{i}-s_{i-1}$ for $1 \leq i \leq k+1$, and $t_{2 i}=s_{i}-b_{i}$ for $1 \leq i \leq k$. (For convenience define $s_{0}=0$ and $b_{k+1}=T$.) ONL's return is $1 /\left(\beta^{t_{2}} \beta^{t_{4}} \cdots \beta^{t_{2 k}}\right)$. OPT's optimal actions, if allowed $k+1$ trades, is to hold during the exact opposite intervals as ONL, i.e., buy at $s_{i}$ and sell at $b_{i+1}$ for $0 \leq i \leq k$. But since it can make at most $k$ trades, its possible course of actions include skipping one of those trades, or making one of the trades 'span across two intervals', e.g., buying at $s_{i}$ and selling at $b_{i+2}$. Thus OPT's return is one of

$$
\begin{gathered}
\alpha^{t_{3}} \alpha^{t_{5}} \cdots \alpha^{t_{2 k+1}}, \alpha^{t_{1}} \alpha^{t_{5}} \cdots \alpha^{t_{2 k+1}}, \ldots, \alpha^{t_{1}} \alpha^{t_{3}} \cdots \alpha^{t_{2 k-1}}, \\
\alpha^{t_{1}} \alpha^{t_{3}} \cdots \alpha^{t_{2 k+1}} / \beta^{t_{2}}, \alpha^{t_{1}} \alpha^{t_{3}} \cdots \alpha^{t_{2 k+1}} / \beta^{t_{4}}, \ldots, \alpha^{t_{1}} \alpha^{t_{3}} \cdots \alpha^{t_{2 k+1}} / \beta^{t_{2 k}} .
\end{gathered}
$$

To attain the worst competitive ratio, these ratios should be equal, which means $t_{1}=t_{3}=\cdots=t_{2 k+1}$ and $t_{2}=t_{4}=\cdots=t_{2 k}$. This further implies $\alpha^{k t_{1}}=\alpha^{(k+1) t_{1}} / \beta^{t_{2}}$, which gives $\alpha^{t_{1}}=\beta^{t_{2}}$. Together with $t_{1}+\cdots+t_{2 k+1}=$ $(k+1) t_{1}+k t_{2}=T$, this gives

$$
t_{1}=\frac{\log \beta}{(k+1) \log \beta+k \log \alpha} T, \quad t_{2}=\frac{\log \alpha}{(k+1) \log \beta+k \log \alpha} T
$$

The competitive ratio is then $\alpha^{k t_{1}} /\left(1 / \beta^{k t_{2}}\right)=\alpha^{2 k t_{1}}=\alpha^{T(2 k \log \beta) /((k+1) \log \beta+k \log \alpha)}$.

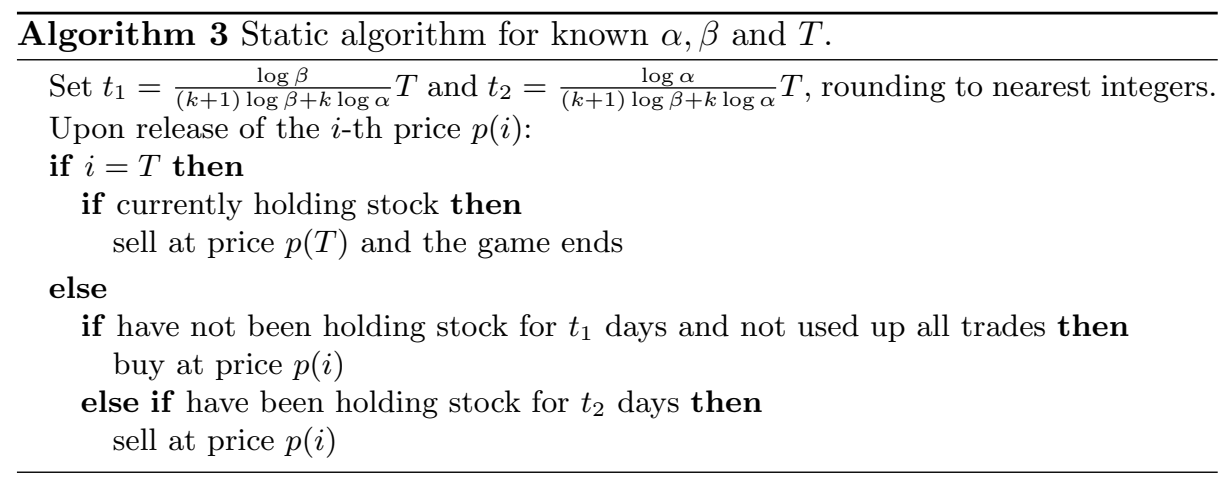

Theorem 7. Algorithm 3 has competitive ratio $\alpha^{T(2 k \log \beta) /((k+1) \log \beta+k \log \alpha)}$. 
Algorithm 3 may feel unnatural since it does not depend on the price sequence at all (this is called 'static' in [1]). But we prove that the following variation of the algorithm has the same competitive ratio: it sells only when the current price falls below $h / \beta^{t_{2}}$ where $h$ is the highest price seen since the last purchase. This coincides with the 'stop loss' strategy very common in real trading (more precisely 'trailing stop' [6] where the stop loss limit is not fixed but tracks the highest price seen thus far, to potentially capture the most profit).

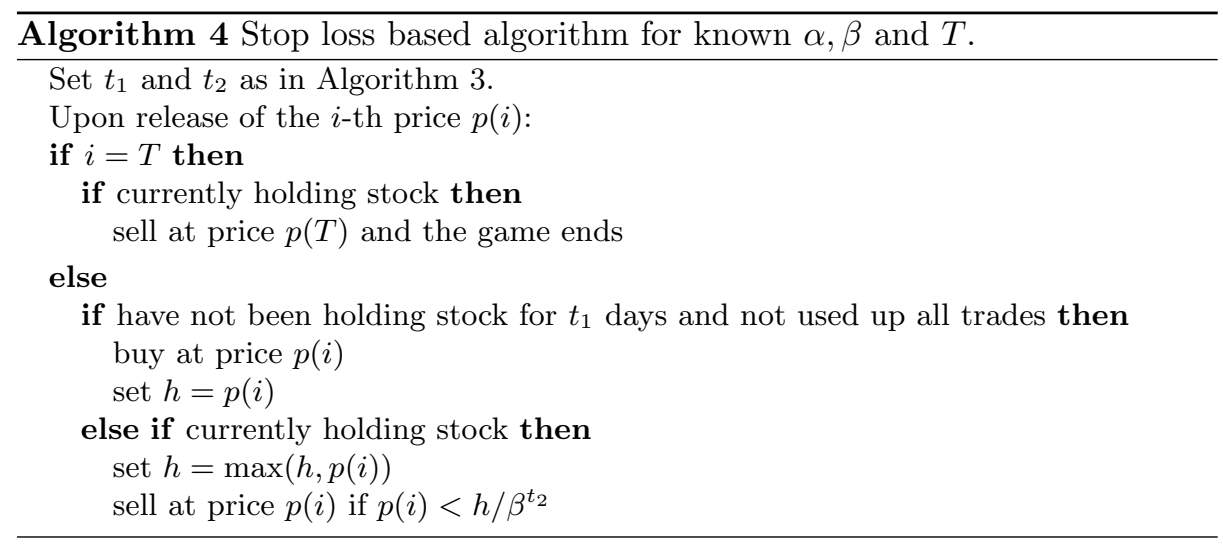

Theorem 8. Algorithm 4 has competitive ratio $\alpha^{T(2 k \log \beta) /((k+1) \log \beta+k \log \alpha)}$.

Proof. (Sketch) Recall that $\alpha^{t_{1}}=\beta^{t_{2}}$. Let $r$ denote this common value, and the competitive ratio we want to prove is then $r^{2 k}$. Our approach is to partition the time horizon so that in each partition $x$ trades in OPT are associated to $y$ trades in ONL such that the ratio between their gains is at most $r^{x+y}$. Since in total they make at most $2 k$ trades, this proves the theorem.

Denote by $\left(b_{i}, s_{i}\right)$ ONL's $i$-th trade. Let $H_{i}=\left[b_{i}, s_{i}\right]$ (a closed time interval) and $N_{i}=\left(s_{i-1}, b_{i}\right)$ (an open interval). Let $h_{i}$ be the highest price during $H_{i}$. We can show the following properties:

(1) For any two days $x$ and $y$ in the same $H_{i}$, where $x<y$, we have $p(y) \geq$ $p(x) / r$. As a direct consequence, $p\left(s_{i}\right) \geq h_{i} / r$.

(2) Without loss of generality we can assume $p\left(b_{i+1}\right)=p\left(s_{i}\right) r$.

(3) OPT would not buy or sell strictly within any $N_{i}$.

Consider an OPT trade $\left(b^{*}, s^{*}\right)$. Suppose $b^{*}$ falls within $H_{u}$ and $s^{*}$ falls within $H_{v}$, where $v \geq u$. Its gain is

$$
\frac{p\left(s^{*}\right)}{p\left(b^{*}\right)} \leq \frac{h_{v}}{p\left(b_{u}\right) / r} \leq \frac{p\left(s_{v}\right) r}{p\left(b_{u}\right) / r}=\frac{p\left(s_{v}\right)}{p\left(b_{u}\right)} r^{2}
$$


where the inequalities are due to (1). Then

$$
\begin{aligned}
& \frac{p\left(s^{*}\right)}{p\left(b^{*}\right)} \leq \frac{p\left(s_{v}\right)}{p\left(b_{v}\right)} \frac{p\left(b_{v}\right)}{p\left(s_{v-1}\right)} \frac{p\left(s_{v-1}\right)}{p\left(b_{v-1}\right)} \frac{p\left(b_{v-1}\right)}{p\left(s_{v-2}\right)} \ldots \frac{p\left(s_{u+1}\right)}{p\left(b_{u+1}\right)} \frac{p\left(b_{u+1}\right)}{p\left(s_{u}\right)} \frac{p\left(s_{u}\right)}{p\left(b_{u}\right)} r^{2} \\
& =\frac{p\left(s_{v}\right)}{p\left(b_{v}\right)} r \frac{p\left(s_{v-1}\right)}{p\left(b_{v-1}\right)} r \ldots r \frac{p\left(s_{u}\right)}{p\left(b_{u}\right)} r^{2}=\frac{p\left(s_{v}\right)}{p\left(b_{v}\right)} \frac{p\left(s_{v-1}\right)}{p\left(b_{v-1}\right)} \ldots \frac{p\left(s_{u}\right)}{p\left(b_{u}\right)} r^{v-u+2}
\end{aligned}
$$

due to (2). If no other OPT trade sells during $H_{u}$ or buys during $H_{v}$, we can associate this $x=1$ OPT trade with these $y=v-u+1$ ONL trades $\left(b_{u}, s_{u}\right), \ldots,\left(b_{v}, s_{v}\right)$. The ratio between their gains is $r^{v-u+2}=r^{1+y}$. Now suppose there are two OPT trades $\left(b^{*}, s^{*}\right)$ and $\left(b^{* *}, s^{* *}\right)$ such that $s^{*}$ and $b^{* *}$ are in the same $H_{i}$. Then $\left(p\left(s^{* *}\right) / p\left(b^{* *}\right)\right)\left(p\left(s^{*}\right) / p\left(b^{*}\right)\right) \leq\left(p\left(s^{* *}\right) / p\left(b^{*}\right)\right) r$ due to (1), and by losing a factor of $r$ we can replace the two OPT trades with one $\left(b^{*}, s^{* *}\right)$. By repeatedly applying the argument, we can partition the time horizon into disjoint parts, where in each part $x$ OPT trades have been merged into one, and this merged trade buys during some $H_{u}$ and sells during some $H_{v}$, and no other OPT trade sells at $H_{u}$ or buys at $H_{v}$. The ratio between the gains, after considering the loss due to merging the OPT trades, is $r^{1+y} r^{x-1}=r^{x+y}$.

\section{References}

1. G.-H. Chen, M.-Y. Kao, Y.-D. Lyuu, and H.-K. Wong. Optimal buy-and-hold strategies for financial markets with bounded daily returns. SIAM Journal on Computing 31(2), 447-459, 2001.

2. F. Y. L. Chin, B. Fu, J. Guo, S. Han, J. Hu, M. Jiang, G. Lin, H. F. Ting, L. Zhang, Y. Zhang, and D. Zhou. Competitive algorithms for unbounded one-way trading. Theoretical Computer Science 607(1), 35-48, 2015.

3. J. Clemente, J. Hromkovic, D. Komm, and C. Kudahl. Advice complexity of the online search problem. Proc. 27th International Workshop on Combinatorial Algorithms (IWOCA), 203-212, 2016.

4. P. Damaschke, P. H. Ha, and P. Tsigas. Online search with time-varying price bounds. Algorithmica 55, 619-642, 2009.

5. R. El-Yaniv, A. Fiat, R. M. Karp, and G. Turpin. Optimal search and one-way trading online algorithms. Algorithmica 30(1), 101-139, 2001.

6. P. W. Glynn and D. L. Iglehart. Trading securities using trailing stops. Management Science 41(6), 1096-1106, 1995.

7. J. Lorenz, K. Panagiotou, and A. Steger. Optimal algorithms for $k$-search with application in option pricing. Algorithmica 55(2), 311-328, 2009.

8. E. Mohr, I. Ahmed, and G. Schmidt. Online algorithms for conversion problems: a survey. Surveys in Operations Research and Management Science 19, 87-104, 2014.

9. G. Schmidt, E. Mohr, and M. Kersch. Experimental analysis of an online trading algorithm. Electronic Notes in Discrete Mathematics 36, 519-526, 2010.

10. P. Schroeder, G. Schmidt and I. Kacem. Optimal on-line algorithms for bidirectional nonpreemptive conversion with interrelated conversion rates. Proc. 4th IEEE Conf. on Control, Decision and Information Technology, 28-33, 2016.

11. W. Zhang, Y. Xu, F. Zheng, and Y. Dong. Optimal algorithms for online time series search and one-way trading with interrelated prices. Journal of Combinatorial Optimization 23, 159-166, 2012. 